\title{
A NOTE ON EMBEDDING CERTAIN BERNOULLI SEQUENCES IN MARKED POISSON PROCESSES
}

\author{
LARS HOLST, ${ }^{*}$ Royal Institute of Technology
}

\begin{abstract}
A sequence of independent Bernoulli random variables with success probabilities $a /(a+$ $b+k-1), k=1,2,3, \ldots$, is embedded in a marked Poisson process with intensity 1 . Using this, conditional Poisson limits follow for counts of failure strings.

Keywords: Bernoulli trial; conditional limit theorem; Poisson limit; random permutation; record; sums of indicators

2000 Mathematics Subject Classification: Primary 60C05

Secondary 60K99
\end{abstract}

\section{Introduction}

Inspired by Huffer et al. (2008) we construct in this note an embedding in a marked Poisson process of a sequence of independent Bernoulli random variables with success probabilities $a /(a+b+k-1), k=1,2,3, \ldots$ From the embedding, conditional Poisson limit distributions follow for the number of $d$-strings, that is, subsequent successes interrupted by $d-1$ failures in the sequence. A special case is the Poisson limits for the number of small cycles in a random permutation biased by the number of cycles.

Other methods have previously been used to obtain such limits; see Arratia et al. (2003), Holst (2007), Holst (2008), Huffer et al. (2008), and the references therein. The embedding technique gives much more concise and transparent derivations and a better understanding of why the Poisson limits occur in such cases.

\section{The embedding}

Let $P, Z_{1}, Z_{2}, Z_{3}, \ldots$ be independent random variables, where the $Z$ s are exponential with mean 1 and $0<P \leq 1$. The waiting time for a $Z$ to exceed $\log (1 / P)$ is

$$
L_{0}=\min \left\{k: Z_{k}>\log \left(\frac{1}{P}\right)\right\},
$$

having the following conditional geometric distribution:

$$
\mathrm{P}\left(L_{0}=\ell \mid P=p\right)=(1-p)^{\ell-1} p, \quad \ell=1,2, \ldots
$$

By the lack of memory property of the exponential distribution, the excess $X_{1}=Z_{L_{0}}-\log (1 / P)$ is exponentially distributed with mean 1 and independent of $\left(P, L_{0}\right)$. Set $T_{1}=X_{1}$.

For $a>0$, the waiting time

$$
L_{1}=\min \left\{k>L_{0}: Z_{k}>\log \left(\frac{1}{P}\right)+\frac{T_{1}}{a}\right\}-L_{0}
$$

Received 27 August 2008; revision received 28 October 2008.

* Postal address: Department of Mathematics, Royal Institute of Technology, SE-100 44 Stockholm, Sweden.

Email address: lholst@math.kth.se 
has the conditional distribution

$$
\mathrm{P}\left(L_{1}=\ell \mid P=p, T_{1}=t\right)=\left(1-p \mathrm{e}^{-t / a}\right)^{\ell-1} p \mathrm{e}^{-t / a}, \quad \ell=1,2, \ldots
$$

The excess $X_{2}=Z_{L_{0}+L_{1}}-\log (1 / P)-T_{1} / a$ is exponentially distributed with mean 1 and independent of $\left(P, L_{0}, L_{1}, T_{1}\right)$. Set $T_{2}=T_{1}+X_{2}$.

Analogously, the waiting time $L_{2}$ for the next $Z$ to exceed $\log (1 / P)+T_{2} / a$ is geometric as above and the excess $X_{3}$ is exponential with mean 1 and independent of $\left(P, L_{0}, L_{1}, L_{2}, T_{1}, T_{2}\right)$. Set $T_{3}=T_{2}+X_{3}$.

In the same way, define the waiting times $L_{3}, L_{4}, \ldots$, the excesses $X_{4}, X_{5}, \ldots$, and the random variables $T_{4}, T_{5}, \ldots$ The sequence of 'records', $T_{1}, T_{2}, T_{3}, \ldots$, is a Poisson process with intensity 1 . Conditional on $P=p,\left\{\left(T_{i}, L_{i}\right), i=1,2,3, \ldots\right\}$ is a marked Poisson process with the marking distribution

$$
\mathrm{P}\left(L_{i}=\ell \mid P=p, T_{i}=t\right)=\left(1-p \mathrm{e}^{-t / a}\right)^{\ell-1} p \mathrm{e}^{-t / a}, \quad \ell=1,2, \ldots
$$

To indicate the times for the records, we introduce the Bernoulli random variables $I_{k}=1$ if $k \in\left\{L_{0}, L_{0}+L_{1}, L_{0}+L_{1}+L_{2}, \ldots\right\}$, otherwise $I_{k}=0$. For $P \equiv 1$ and $a=1$, the $I$ s indicate ordinary records among the $Z$ s. Rényi's theorem shows that these indicators are independent with $\mathrm{P}\left(I_{n}=1\right)=1 / n$. The theorem below generalizes this well-known result.

We say that a random variable $P$ with density

$$
f(p)=\frac{\Gamma(a+b)}{\Gamma(a) \Gamma(b)} p^{a-1}(1-p)^{b-1}, \quad 0<p<1,
$$

is $\operatorname{Beta}(a, b)$, where $a>0$ and $b>0 ; \operatorname{Beta}(a, 0)$ is interpreted as $P \equiv 1$. Recall that

$$
\mathrm{E}\left(P^{k}(1-P)^{n-k}\right)=\frac{a^{\bar{k}} b^{\overline{n-k}}}{(a+b)^{\bar{n}}} .
$$

We use the notation $s^{\bar{n}}=s(s+1) \cdots(s+n-1)$ for rising factorials.

Theorem 2.1. Let $P$ be $\operatorname{Beta}(a, b), a>0$ and $b \geq 0$. Then the record indicators, $I_{1}, I_{2}, I_{3}, \ldots$, are independent random variables with $\mathrm{P}\left(I_{n}=1\right)=a /(a+b+n-1)$.

Proof. We give a proof for the case in which $b>0$. The proof is easily modified for $b=0$, that is, for $P \equiv 1$.

Consider $I_{1}, I_{2}, \ldots, I_{n}$. We have

$$
\mathrm{P}\left(I_{1}=\cdots=I_{n}=0\right)=\mathrm{P}\left(L_{0}>n\right)=\mathrm{E}\left((1-P)^{n}\right)=\frac{b^{\bar{n}}}{(a+b)^{\bar{n}}}
$$

and

$$
\mathrm{P}\left(I_{1}=\cdots=I_{n-1}=0, I_{n}=1\right)=\mathrm{P}\left(L_{0}=n\right)=\mathrm{E}\left((1-P)^{n-1} P\right)=\frac{a b^{\overline{n-1}}}{(a+b)^{\bar{n}}} .
$$


Changing variables and integrating by parts we obtain, for $1 \leq \ell<n$,

$$
\begin{aligned}
f_{0}(n, a, b, \ell) & =\mathrm{P}\left(L_{0}=\ell, L_{1}>n-\ell\right) \\
& =\mathrm{E}\left((1-P)^{\ell-1} P \int_{0}^{\infty}\left(1-P \mathrm{e}^{-x / a}\right)^{n-\ell} \mathrm{e}^{-x} \mathrm{~d} x\right) \\
& =\mathrm{E}\left((1-P)^{\ell-1} P \int_{0}^{1}(1-P u)^{n-\ell} a u^{a-1} \mathrm{~d} u\right) \\
& =\mathrm{E}\left((1-P)^{n-1} P\right)+(n-\ell) \mathrm{E}\left((1-P)^{\ell-1} P^{2} \int_{0}^{1}(1-P u)^{n-\ell-1} u^{a} \mathrm{~d} u\right) \\
& =\frac{a b^{\overline{n-1}}}{(a+b)^{\bar{n}}}+\frac{a}{a+b} \frac{n-\ell}{a+1} f_{0}(n-1, a+1, b, \ell) .
\end{aligned}
$$

Induction proves that

$$
f_{0}(n, a, b, \ell)=\mathrm{P}\left(L_{0}=\ell, L_{1}>n-\ell\right)=\frac{a b^{\bar{n}}}{(a+b)^{\bar{n}}(b+\ell-1)} .
$$

For $1 \leq \ell_{0}, \ldots, \ell_{j}, \ell_{0}+\cdots+\ell_{j} \leq n$, set

$$
f_{j}\left(n, a, b, \ell_{0}, \ldots, \ell_{j}\right)=\mathrm{P}\left(I_{k}=1 \text { if } k \in\left\{\ell_{0}, \ell_{0}+\ell_{1}, \ldots, \ell_{0}+\cdots+\ell_{j}\right\}, \text { else } I_{k}=0\right) .
$$

Changing variables we find that

$$
\begin{aligned}
& f_{j}\left(n, a, b, \ell_{0}, \ldots, \ell_{j}\right) \\
& =\mathrm{P}\left(L_{0}=\ell_{0}, \ldots, L_{j}=\ell_{j}, L_{j+1}>n-\ell_{0}-\cdots-\ell_{j}\right) \\
& =\mathrm{E}\left((1-P)^{\ell_{0}-1} P\right. \\
& \times \int_{0}^{\infty} \cdots \int_{0}^{\infty}\left(1-P \mathrm{e}^{-x_{1} / a}\right)^{\ell_{1}-1} P \mathrm{e}^{-x_{1} / a} \cdots\left(1-P \mathrm{e}^{-\left(x_{1}+\cdots+x_{j}\right) / a}\right)^{\ell_{j}-1} \\
& \times P \mathrm{e}^{-\left(x_{1}+\cdots+x_{j}\right) / a}\left(1-P \mathrm{e}^{-\left(x_{1}+\cdots+x_{j+1}\right) / a}\right)^{n-\ell_{0}-\cdots-\ell_{j}} \\
& \left.\times \mathrm{e}^{-\left(x_{1}+\cdots+x_{j+1}\right)} \mathrm{d} x_{1} \cdots \mathrm{d} x_{j+1}\right) \\
& =\mathrm{E}\left((1-P)^{\ell_{0}-1} P a^{j+1}\right. \\
& \times \int_{0}^{1} \cdots \int_{0}^{1}\left(1-P u_{1}\right)^{\ell_{1}-1} P u_{1} \cdots\left(1-P u_{1} \cdots u_{j}\right)^{\ell_{j}-1} P u_{1} \cdots u_{j} \\
& \left.\times\left(1-P u_{1} \cdots u_{j+1}\right)^{n-\ell_{0}-\cdots-\ell_{j}} u_{1}^{a-1} \cdots u_{j+1}^{a-1} \mathrm{~d} u_{1} \cdots \mathrm{d} u_{j+1}\right) \\
& =\mathrm{E}\left((1-P)^{\ell_{0}-1} P a^{j}\right. \\
& \times \int_{0}^{1} \cdots \int_{0}^{1}\left(1-P u_{1}\right)^{\ell_{1}-1} P u_{1}^{a+j-1} \cdots\left(1-P u_{1} \cdots u_{j}\right)^{\ell_{j}-1} P u_{j}^{a} \\
& \left.\times\left(\int_{0}^{1}\left(1-P u_{1} \cdots u_{j+1}\right)^{n-\ell_{0}-\cdots-\ell_{j}} a u_{j+1}^{a-1} \mathrm{~d} u_{j+1}\right) \mathrm{d} u_{1} \cdots \mathrm{d} u_{j}\right) .
\end{aligned}
$$


Integration by parts gives

$$
\begin{aligned}
\int_{0}^{1}(1- & \left.P u_{1} \cdots u_{j+1}\right)^{n-\ell_{0}-\cdots-\ell_{j}} a u_{j+1}^{a-1} \mathrm{~d} u_{j+1} \\
= & \left(1-P u_{1} \cdots u_{j}\right)^{n-\ell_{0}-\cdots-\ell_{j}} \\
& \quad+\left(n-\ell_{0}-\cdots-\ell_{j}\right) P u_{1} \cdots u_{j} \int_{0}^{1}\left(1-P u_{1} \cdots u_{j+1}\right)^{n-\ell_{0}-\cdots-\ell_{j}-1} u_{j+1}^{a} \mathrm{~d} u_{j+1},
\end{aligned}
$$

implying the recursion

$$
\begin{aligned}
& f_{j}\left(n, a, b, \ell_{0}, \ldots, \ell_{j}\right) \\
& =\frac{a}{a+b} \int_{0}^{1} \frac{\Gamma(a+b+1)}{\Gamma(a+1) \Gamma(b)} p^{a}(1-p)^{b-1}(1-p)^{\ell_{0}-1} p \\
& \times\left(a^{j} \int_{0}^{1} \cdots \int_{0}^{1}\left(1-p u_{1}\right)^{\ell_{1}-1} p u_{1} \cdots\left(1-p u_{1} \cdots u_{j-1}\right)^{\ell_{j-1}-1} p u_{1} \cdots u_{j-1}\right. \\
& \times\left(1-p u_{1} \cdots u_{j}\right)^{n-1-\ell_{0}-\cdots-\ell_{j-1}} u_{1}^{a} \cdots u_{j}^{a} \mathrm{~d} u_{1} \cdots \mathrm{d} u_{j} \\
& +\left(n-\ell_{0}-\cdots-\ell_{j}\right) a^{j} \int_{0}^{1} \cdots \int_{0}^{1}\left(1-p u_{1}\right)^{\ell_{1}-1} p u_{1} \cdots\left(1-p u_{1} \cdots u_{j}\right)^{\ell_{j}-1} \\
& \left.\times p u_{1} \cdots u_{j}\left(1-p u_{1} \cdots u_{j+1}\right)^{n-1-\ell_{0}-\cdots-\ell_{j}} u_{1}^{a} \cdots u_{j+1}^{a} \mathrm{~d} u_{1} \cdots \mathrm{d} u_{j+1}\right) \mathrm{d} p \\
& =\frac{a}{a+b}\left(\frac{a^{j}}{(a+1)^{j}} f_{j-1}\left(n-1, a+1, b, \ell_{0}, \ldots, \ell_{j-1}\right)\right. \\
& \left.+\left(n-\ell_{0}-\cdots-\ell_{j}\right) \frac{a^{j}}{(a+1)^{j+1}} f_{j}\left(n-1, a+1, \ell_{0}, \ldots, \ell_{j}\right)\right) .
\end{aligned}
$$

This is satisfied by

$$
\begin{aligned}
& f_{j}\left(n, a, b, \ell_{0}, \ldots, \ell_{j}\right) \\
& \quad=\mathrm{P}\left(L_{0}=\ell_{0}, \ldots, L_{j}=\ell_{j}, L_{j+1}>n-\ell_{0}-\cdots-\ell_{j}\right) \\
& \quad=\frac{a^{j+1} b^{\bar{n}}}{(a+b)^{\bar{n}}\left(b+\ell_{0}-1\right)\left(b+\ell_{0}+\ell_{1}-1\right) \cdots\left(b+\ell_{0}+\cdots+\ell_{j}-1\right)} .
\end{aligned}
$$

From this, it follows that $I_{1}, I_{2}, I_{3}, \ldots$ are independent Bernoulli random variables with $\mathrm{P}\left(I_{k}=1\right)=a /(a+b+k-1)$.

\section{Poisson limits}

Conditional on $P=p$, the marking theorem in Kingman (1993, Section 5.2) shows that the sequences

$$
\left\{\left(T_{i}, L_{i}=\ell\right), i=1,2, \ldots\right\}, \quad \ell=1,2, \ldots,
$$

are independent marked Poisson processes on the positive real line with intensities

$$
\lambda_{\ell}(t)=\left(1-p \mathrm{e}^{-t / a}\right)^{\ell-1} p \mathrm{e}^{-t / a}, \quad \ell=1,2, \ldots
$$


Thus, the number of $T$ s marked with $\ell, N_{\ell}$, is Poisson with mean

$$
\int_{0}^{\infty} \lambda_{\ell}(t) \mathrm{d} t=\frac{a}{\ell}\left(1-(1-p)^{\ell}\right)
$$

and $N_{1}, N_{2}, \ldots$ are conditionally independent.

Let $I_{1}, I_{2}, I_{3}, \ldots$ be independent Bernoulli variables with success probabilities $a /(a+b+$ $k-1), k=1,2, \ldots$ By the above theorem, such a sequence can be considered as a record indicator in an embedding where $P$ is $\operatorname{Beta}(a, b)$. Consider the number of $d$-strings, that is,

$$
M_{d}=\sum_{k=1}^{\infty} I_{k}\left(1-I_{k+1}\right) \cdots\left(1-I_{k+d-1}\right) I_{k+d},
$$

which, by the embedding, can be identified by $N_{d}$. Hence, conditional on $P=p$, the random variables $M_{1}, M_{2}, \ldots$ are independent Poisson with means as above. This agrees with results in Holst (2007) and Huffer et al. (2008).

For $a=\theta>0$ and $b=0$, the Bernoulli variables above appear in connection with $\theta$-biased random permutations; see Arratia et al. (2003, pp. 95, 96). The counts of different failure strings in $1 I_{2} \cdots I_{n} 1$ correspond to the number of cycles $C_{1}^{(n)}, C_{2}^{(n)}, \ldots, C_{n}^{(n)}$ of sizes $1,2, \ldots, n$ in a $\theta$-biased random permutation of $1,2, \ldots, n$. The limit counts as $n \rightarrow \infty$ for the number of small cycles are given by independent Poisson random variables $M_{1}, M_{2}, \ldots$ with $\mathrm{E}\left(M_{d}\right)=\theta / d$; cf. Arratia et al. (2003, Theorem 5.1).

Finally, consider a sequence of independent indicators, $I_{1} \equiv 1, I_{2}, I_{3}, \ldots$, with $\mathrm{P}\left(I_{k}=1\right)=$ $a /(a+b+k-2), k=2,3, \ldots$, where $b \geq 1$. With $Z$ exponential with mean 1 and independent of $P$, which is $\operatorname{Beta}(a+1, b-1)$, we find that $P^{\prime}=P \mathrm{e}^{-Z / a}$ is $\operatorname{Beta}(a, b)$. Using $P^{\prime}$, we can generate, by the embedding, a sequence $I_{1}^{\prime}, I_{2}^{\prime}, \ldots$ with $\mathrm{P}\left(I_{k}^{\prime}=1\right)=a /(a+b+k-1)$. For $k=2,3, \ldots$, set $I_{k}=I_{k-1}^{\prime}$ with $\mathrm{P}\left(I_{k}=1\right)=a /(a+b+k-2)$. Conditional on $P=p$, the number of $d$-strings in $1 I_{2} I_{3} \ldots$ is a Poisson random variable $M_{d}$ with mean $a\left(1-(1-p)^{d}\right) / d$ and $M_{1}, M_{2}, \ldots$ are independent. This is in agreement with Huffer et al. (2008). For $b<1$, the distribution of $M_{d}$ is not conditional Poisson; see Huffer et al. (2008).

\section{Acknowledgement}

I am grateful to an anonymous referee for suggesting an extension of the original embedding.

\section{References}

Arratia, R., Barbour, A. D. and Tavaré, S. (2003). Logarithmic Combinatorial Structures: A Probabilistic Approach. European Mathematical Society, Zürich.

Holst, L. (2007). Counts of failure strings in certain Bernoulli sequences. J. Appl. Prob. 44, 824-830.

Holst, L. (2008). The number of two consecutive successes in a Hoppe-Pólya urn. J. Appl. Prob. 45, 901-906.

Huffer, F., Sethuraman, J. and Sethuraman, S. (2008). A study of counts of Bernoulli strings via conditional Poisson processes. To appear in Proc. Amer. Math. Soc.

Kingman, J. F. C (1993). Poisson Processes (Oxford Studies Prob. 3). Oxford University Press. 